\title{
Peli1 facilitates virus replication and promotes neuroinflammation during West Nile virus infection
}

\author{
Huanle Luo, ${ }^{1}$ Evandro R. Winkelmann, ${ }^{1}$ Shuang Zhu, ${ }^{2}$ Wenjuan Ru, ${ }^{3}$ Elizabeth Mays, ${ }^{1}$ Jesus A. Silvas, ${ }^{4}$ Lauren L. Vollmer, ${ }^{5}$ \\ Junling Gao, ${ }^{3}$ Bi-Hung Peng, ${ }^{3}$ Nathen E. Bopp, ${ }^{4}$ Courtney Cromer, ${ }^{4}$ Chao Shan, ${ }^{6}$ Guorui Xie, ${ }^{1}$ Guangyu Li, ${ }^{1}$ Robert Tesh, ${ }^{4,7}$ \\ Vsevolod L. Popov, ${ }^{4,7}$ Pei-Yong Shi, ${ }^{6,7}$ Shao-Cong Sun, ${ }^{8}$ Ping Wu, ${ }^{3,7}$ Robyn S. Klein, ${ }^{5}$ Shao-Jun Tang, ${ }^{3,7}$ Wenbo Zhang, ${ }^{2,3,7}$ \\ Patricia V. Aguilar, ${ }^{4,7}$ and Tian Wang ${ }^{1,4,7}$
}

'Department of Microbiology and Immunology, ${ }^{1}$ Department of Ophthalmology and Visual Sciences, ${ }^{3}$ Department of Neuroscience, Cell Biology and Anatomy, and ${ }^{4}$ Department of Pathology, University of Texas Medical Branch (UTMB), Galveston, Texas, USA. 'DDepartment of Medicine, Washington University School of Medicine, St. Louis, Missouri, USA. ${ }^{6}$ Department of Biochemistry and Molecular Biology, and ${ }^{7}$ Institute for Human Infections and Immunity, UTMB, Galveston, Texas, USA. ${ }^{8}$ Department of Immunology, The University of Texas MD Anderson Cancer Center, Houston, Texas, USA.

\begin{abstract}
The E3 ubiquitin ligase Pellino 1 (Peli1) is a microglia-specific mediator of autoimmune encephalomyelitis. Its role in neurotropic flavivirus infection is largely unknown. Here, we report that mice deficient in Peli1 (Peli1 ${ }^{-/}$) were more resistant to lethal West Nile virus (WNV) infection and exhibited reduced viral loads in tissues and attenuated brain inflammation. Peli1 mediates chemokine and proinflammatory cytokine production in microglia and promotes $\mathrm{T}$ cell and macrophage infiltration into the CNS. Unexpectedly, Peli1 was required for WNV entry and replication in mouse macrophages and mouse and human neurons and microglia. It was also highly expressed on WNV-infected neurons and adjacent inflammatory cells from postmortem patients who died of acute WNV encephalitis. WNV passaged in Peli1/- macrophages or neurons induced a lower viral load and impaired activation in WT microglia and thereby reduced lethality in mice. Smaducin-6, which blocks interactions between Peli1 and IRAK1, RIP1, and IKK , did not inhibit WNV-triggered microglia activation. Collectively, our findings suggest a nonimmune regulatory role for Peli1 in promoting microglia activation during WNV infection and identify a potentially novel host factor for flavivirus cell entry and replication.
\end{abstract}

\section{Introduction}

Pellino 1 (Peli1), an E3 ubiquitin ligase, is an important regulator in innate and adaptive immunity. It is essential for NF- $\mathrm{KB}$ activation induced by TIR domain-containing, adapter-inducing IFN- $\beta / T L R-$ mediated (TRIF/TLR) signaling in innate immune cells such as macrophages and DCs (1). In microglial cells, in which Peli1 is predominantly expressed in the brain, Peli1 regulates TLR/MyD88 signaling by promoting the degradation of TNF receptor-associated factor 3 (Traf3), a process which leads to microglia activation during the course of induction of experimental autoimmune encephalomyelitis (EAE) (2). Peli1 is also implicated in the regulation of adaptive immune cell functions. For example, it is a critical factor in the maintenance of peripheral $\mathrm{T}$ cell tolerance by regulating c-Rel via its K48 ubiquitination (3). Peli1 upregulation also plays a role in B cell lymphoma tumorigenesis (4).

West Nile virus (WNV), a mosquito-borne, single-stranded flavivirus that caused outbreaks in Asia, Europe, and Australia, has been the leading cause of viral encephalitis in the United States for more than a decade $(5,6)$. The features of acute illness range from WN fever to neuroinvasive conditions, including meningitis,

Authorship note: $\mathrm{HL}$ and ERW contributed equally to this work. Conflict of interest: The authors have declared that no conflict of interest exists. Submitted: January 22, 2018; Accepted: August 7, 2018.

Reference information: J Clin Invest. 2018;128(11):4980-4991. https://doi.org/10.1172/JCI99902. encephalitis, acute flaccid paralysis, and death (7). In addition, up to $50 \%$ of convalescent WNV patients have been reported to have long-term neurological sequelae or chronic kidney disease (8-16). Currently, there is no specific therapeutic agent for the treatment of WNV infection, and an approved vaccine is not available for humans. Studies in animal models suggest that WNV-induced CNS disease is caused by neuronal degeneration, a direct result of viral infection, and/or by bystander damage from the immune response to the pathogen in the CNS $(17,18)$ that is induced by infiltrating inflammatory cells and CNS-resident cells, including microglia/macrophages, neutrophils, and lymphocytes (19-23). Microglial cells, the resident macrophages of the CNS that express various pattern recognition receptors (PRRs), become activated and produce innate proinflammatory molecules upon encountering microbial infection. Microglia activation in the CNS is the hallmark of acute WNV infection $(24,25)$. It was also shown to drive neuronal and synaptic loss, which together contributes to memory impairment in WNV-induced chronic cognitive sequelae (26). The underlying immune mechanisms of microglia activation are not clearly understood. Here, we hypothesize that Peli1 mediates the activation of microglia and promotes neuroinflammation during lethal WNV infection. Unexpectedly, we found that Peli1 is required for WNV entry and replication in both peripheral myeloid cells and CNS-resident cells. In particular, we found that defective WNV replication in Peli1-deficient microglia and neurons directly contributes to attenuated neuroinflammation in the CNS and ultimately decreases host susceptibility to lethal encephalitis. 

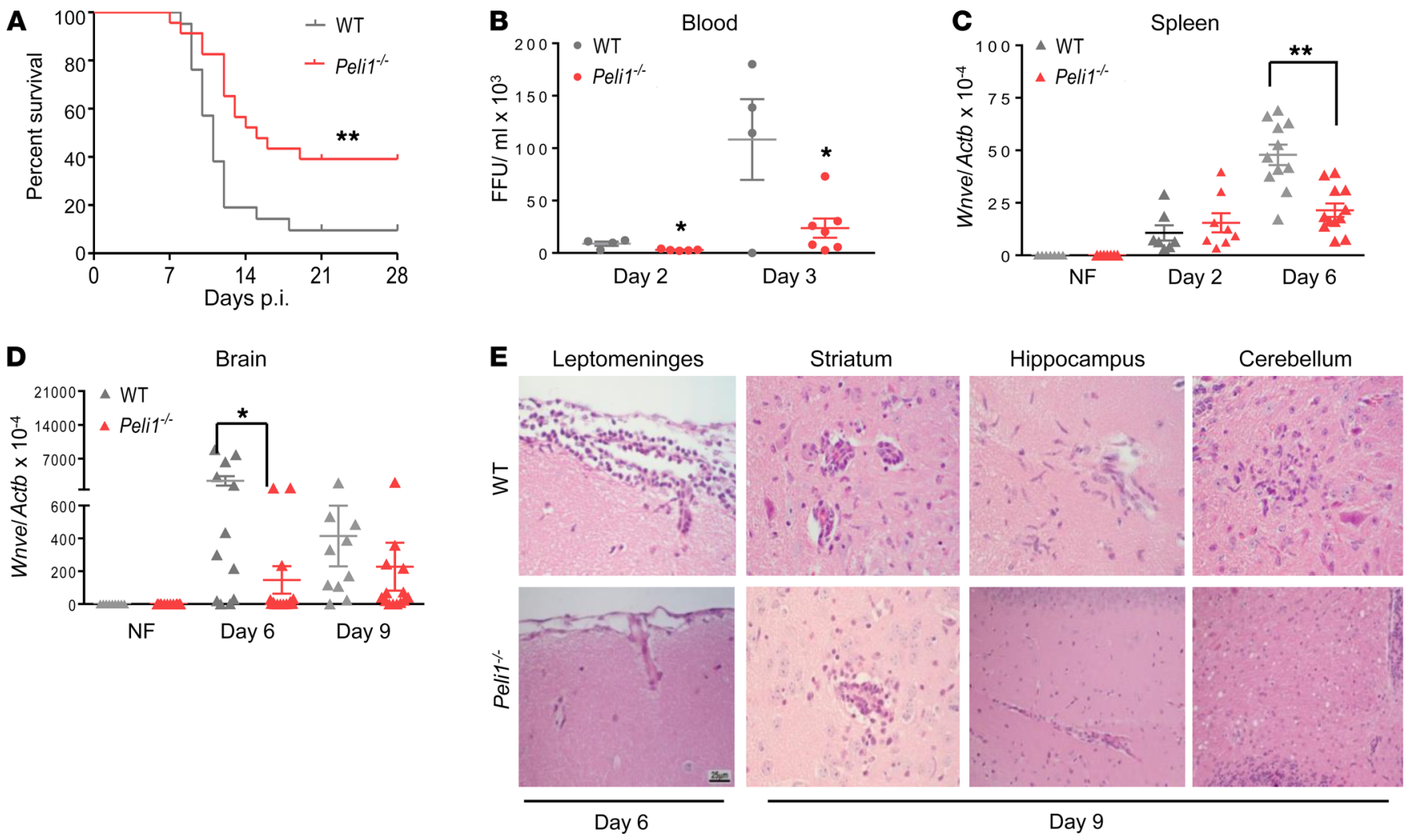

Figure 1. Peli1 ${ }^{-1-}$ mice are more resistant to lethal WNV infection. (A) Survival of WT and Peli ${ }^{-1-}$ mice after i.p. injection with WNV $385-99 . n=21$ WT mice; $n=23$ and Peli1 ${ }^{-1-}$ mice. ${ }^{* *} P<0.01$ compared with the WT group (log-rank test). (B) Viremia was determined by FFA on days 2 and 3 p.i. Data are presented as the mean \pm SEM $(n=3-6)$ of samples collected from 1 representative experiment of 3 similar experiments. (C and $\mathbf{D})$ Viral loads in the spleens and brains of infected and noninfected (NF) mice were determined by qPCR. Data are presented as the mean \pm SEM $(n=7-12)$ and were collected from 3 independent experiments. (B-D) ${ }^{*} P<0.05$ and ${ }^{*} P<0.01$ compared with the WT group (unpaired, 2-tailed Student's $t$ test). (E) Representative H\&Estained images are of brains collected from 4 or 5 WNV-infected mice per group at the indicated time points. Scale bar: $25 \mu \mathrm{m}$. Original magnification, $\times 20$.

\section{Results}

Peli1 ${ }^{--}$mice are more resistant to systemic WNV infection. Peli1 promotes microglia-mediated brain inflammation in the course of EAE induction (2). To investigate the role of Peli1 in WNV encephalitis, we infected WT and Peli1 ${ }^{-/}$mice i.p. with 100 focus-forming units (FFU) of the WNV 385-99 strain and monitored them daily for survival (Figure 1A). Peli1 ${ }^{-/}$mice (39\% survival) were more resistant to WNV infection than were WT controls (9.5\% survival). To further understand the viral pathogenesis, we measured viral burden in the peripheral organs and brain. Peli1 ${ }^{-1-}$ mice had lower viremia on days 2 and 3 post infection (p.i.) and decreased splenic viral loads on day 6 compared with WT mice (Figure 1, B and C). WNV crosses the blood-brain barrier (BBB) and infects the CNS around day 3 in mice (25). Viral RNA levels in Peli1 ${ }^{-/}$mouse brains were more than 15 -fold lower than those of WT mice on day 6 p.i. (Figure 1D). This trend continued but became insignificant by day 9 , when both groups of mice started to succumb to lethal WNV infection. On day 6 p.i., we noted meningitis (inflammation of the leptomeninge) in WT mice, but not in Peli1 $^{-/-}$mice (Figure 1E). Inflammation further spread to the brain parenchyma (encephalitis), as seen on day 9 in the striatum, hippocampus, and cerebellum in both groups of mice. We found that encephalitis, particularly perivascular cuffing and microglia activation (cells with elongated nuclei), was much more extensive in the WT mice.
Thus, CNS inflammation started earlier and was more severe in WNV-infected WT mice than in Peli1 ${ }^{-/}$mice. No histopathological differences were noted in naive mouse brains between the WT and $\mathrm{Peli1}^{-/-}$groups (Supplemental Figure 1; supplemental material available online with this article; https://doi.org/10.1172/ JCI99902DS1).

Peli1 $^{-1}$ mice exhibit impaired innate cytokine production, but modestly enhanced adaptive immune responses in the periphery. Peli1 is known to facilitate TRIF-dependent TLR signaling and proinflammatory cytokine production (1). Following WNV infection, Peli1 expression was increased in the blood of WT mice (Supplemental Figure 2A). The RNA levels of IFN- $\alpha$ (Ifna) and IFN- $\beta$ (Ifnb) on day 6, IL-6 (Il6) and TNF- $\alpha$ (Tnfa) on days 3 and 6, and Il12 on day 3 were all diminished in Peli1 ${ }^{-/-}$mice (Supplemental Figure 2B). Blood plasma protein levels of IL-1 $\beta$ and IL-10 were also reduced in $\mathrm{Peli1}^{-/-}$mice (Supplemental Table 1), though no differences were noted in plasma IFN- $\gamma$ or IL-17 levels between the 2 groups of mice. To study the adaptive immune responses in the periphery, we first measured antibody production in the blood. WNV-specific IgM responses were modestly enhanced in Peli1 ${ }^{-/-}$ mice on days 3 and 9 p.i. (Supplemental Figure 2C). WNV-specific IgG responses were similar between the 2 groups of mice (Supplemental Figure 2D). Peli1 is also known to negatively regulate $\mathrm{T}$ cell signaling (3). We next collected splenic tissues from naive and 
A

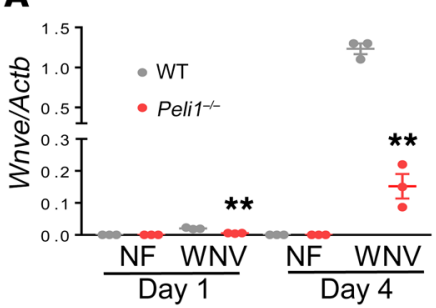

B

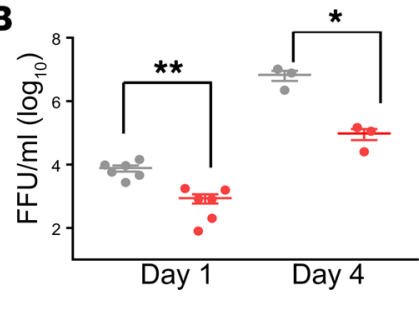

C

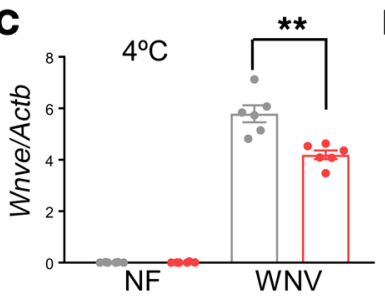

D

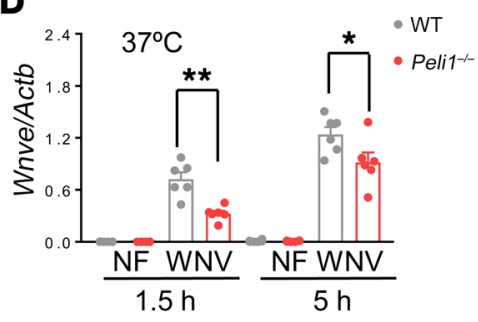

E

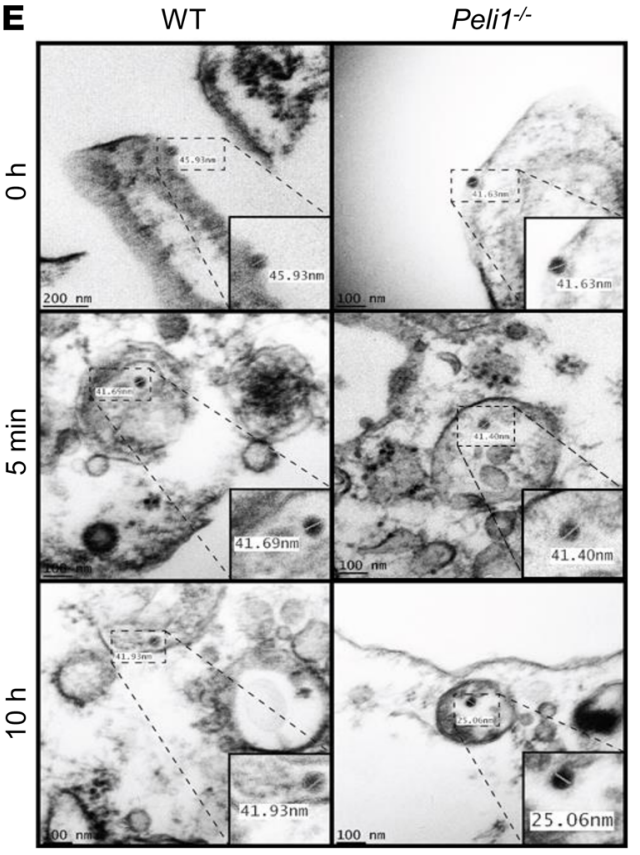

$\mathbf{F}$

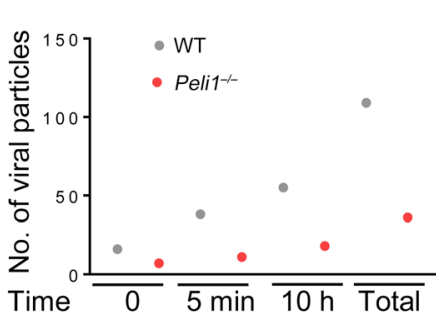

H

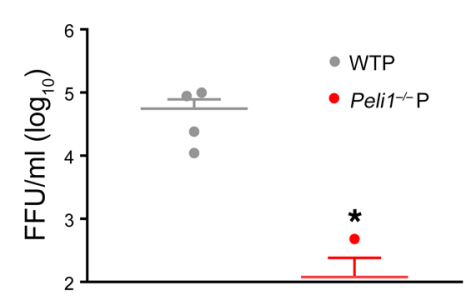

G

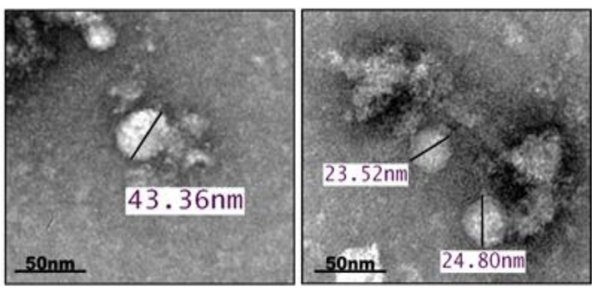

I

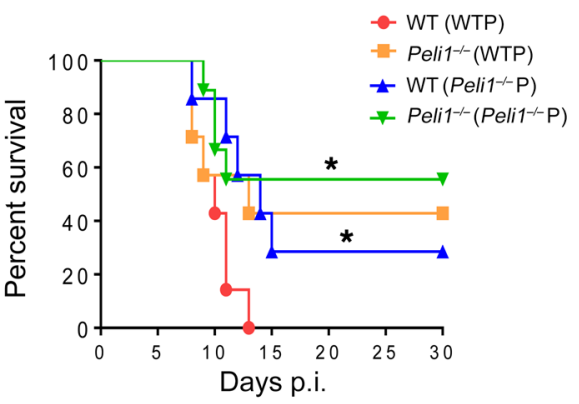

Figure 2. Peli1 facilitates WNV replication in macrophages and promotes high mortality in vivo. (A and B) The viral load of WNV-infected macrophages was measured by qPCR (A) and FFA (B). Data are representative of 5 similar experiments and are presented as the mean \pm SEM $(n=3-6)$. (C and $\mathbf{D})$ Macrophages were infected with WNV for 1 hour at $4^{\circ} \mathrm{C}$, washed, and then collected to measure intracellular viral RNA by qPCR in the attachment assay (C). For virus entry $(\mathbf{D})$, cells were subsequently resuspended in medium and incubated at $37^{\circ} \mathrm{C}$. At the indicated time points, cells were washed to determine intracellular viral RNA levels $(n=6)$. ( $(\mathbf{E}$ and $\mathbf{F})$ Thin-section transmission electron micrographs of WNV-infected macrophages. (E) Viral particles were observed at the plasma membrane at 0 minutes, in small, uncoated vesicles at 5 minutes, and in double-membrane vesicles at 10 hours. (F) Quantitation of 10 fields of view of ultrathin sections. (C) Negative staining micrographs of WNV. Virions ranged in size from $41 \mathrm{~nm}$ to $47 \mathrm{~nm}$ and $23.5 \mathrm{~nm}$ to $39.5 \mathrm{~nm}$ in supernatants of WT and Pelit ${ }^{-1-}$ macrophages on day 4 p.i., respectively. (H) WT macrophages were infected at a MOI of 0.02 with viruses passaged in WT (WTP) and Pelit ${ }^{-1-}\left(\right.$ Peli $\left.{ }^{-1-P}\right)$ macrophages. The viral load was measured by FFA on day 4 p.i. Data are representative of 2 similar experiments and are presented as the mean \pm SEM $(n=4)$. Data in $\mathbf{A}-\mathbf{D}$ and $\mathbf{H}$ were analyzed by unpaired, 2-tailed Student's $t$ test. ${ }^{*} P<0.05$ and ${ }^{* *} P<0.01$ compared with the WT group. (I) Survival rates of mice injected i.p. with 100 FFU WNV passaged in WT macrophages and Peli1 ${ }^{-1-}$ macrophages $(n=7-9)$. ${ }^{*} P<0.05$ compared with WT mice infected with WT macrophages (log-rank test).

WNV-infected WT and Peli1 ${ }^{-/}$mice. We found no necrosis in any of the splenic sections examined. We noted a trend toward white pulp expansion resulting from germinal center proliferation in WT mice on days 3 and 6 p.i., but this returned to levels similar to those in naive mice by day 9; in Peli1 ${ }^{--}$mice, the expansion was continuously enhanced (Supplemental Figure 2, E and F). On day 7, both $\mathrm{CD}^{+}$and $\mathrm{CD} 8^{+}$splenic $\mathrm{T}$ cells from Peli1 ${ }^{--}$mice produced more IFN- $\gamma$ than did those from WT mice upon ex vivo restimulation with WNV-specific peptides. CD $4^{+} \mathrm{T}$ cells from Peli1 ${ }^{-/}$mice also induced higher levels of IL- 6 and IL-10 production (Supplemental Figure 2, $\mathrm{G}$ and $\mathrm{H}$ ).

Peli1 is required for WNV entry and viral replication in macrophages and DCs, which further activates innate cytokine responses. Macrophages and DCs are innate immune cells expressing many
PRRs, and both cell types are permissive to WNV infection in the peripheral organs (27). To study Peli1-mediated innate immune responses upon WNV infection, we first measured viral loads in these 2 cell types by quantitative PCR (qPCR) and focus-forming assays (FFAs) and found that both viral RNA levels and viral titers were significantly diminished in primary myeloid Peli1 ${ }^{-1-}$ macrophages and DCs compared with WT controls (Figure 2, A and B, and Supplemental Figure 3, A and B). We next performed virus attachment and entry assays to determine Peli1 involvement in the WNV replication cycle. Macrophages from mice of both groups were incubated with WNV $(\mathrm{MOI}=3$ or 10$)$ at $4^{\circ} \mathrm{C}$ for 1 hour, allowing the virus to attach to the cell surface. After 1 hour of incubation, the cells were washed to remove unattached virus, and the amounts of viruses that had attached to the cell surface 
A

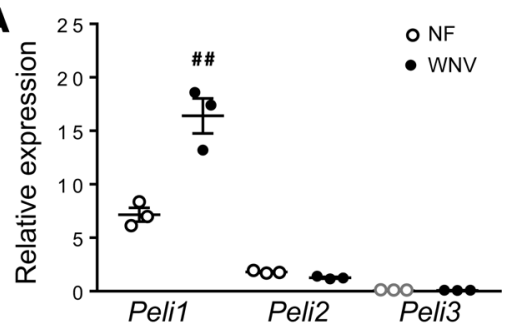

B

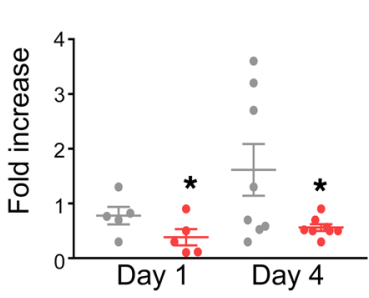

C

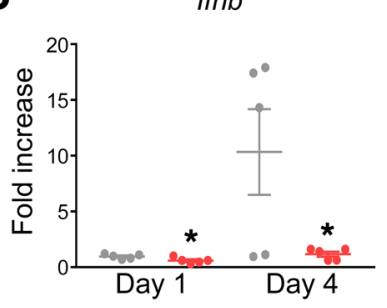

D

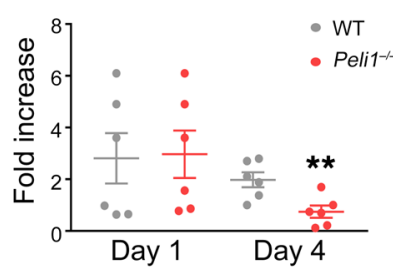

E

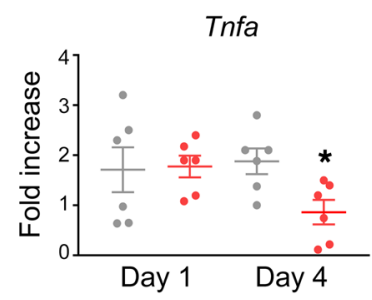

F

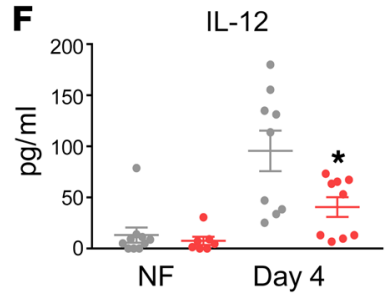

G

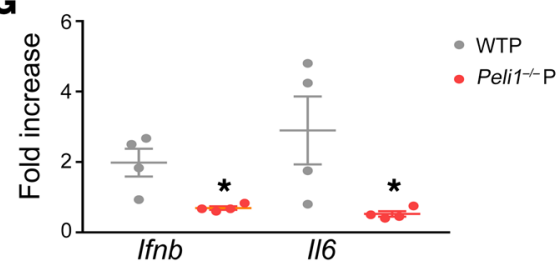

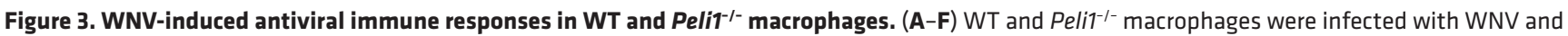
harvested at the indicated time points. (A) Peli1, Peli2, and Peliz RNA levels in WT macrophages were measured on day 4 p.i. Data are representative of 3 similar experiments and are presented as the mean $\pm \operatorname{SEM}(n=3)$. ${ }^{\#} P<0.01$ compared with the noninfected group (unpaired, 2-tailed Student's $t$ test). (B-F) Cytokine RNA or protein levels were measured at the indicated time points by qPCR or Bio-Plex, respectively. Data represent the mean \pm SEM of 5 to 10 samples collected from 2 independent experiments. ${ }^{*} P<0.05$ and ${ }^{* *} P<0.01$ compared with the WT group. (C) WT macrophages were infected at a $\mathrm{MOI}$ of 0.02 with WNV passaged in WT or Peli ${ }^{-1-}$ macrophages. On day 4 p.i., cytokine production was determined by qPCR. Data are presented as the fold increase compared with mock-infected cells (mean \pm SEM) and are representative of 2 similar experiments $\left(n=4\right.$ per group). ${ }^{*} P<0.05$ compared with the WTP group (unpaired, 2-tailed Student's $t$ test).

were measured by qPCR. We noted that Peli1 ${ }^{-/-}$macrophages had $28 \%$ less virus attachment than did the WT cells (Figure 2C). Cells were further incubated at $37^{\circ} \mathrm{C}$ to initiate viral entry. At 1.5 and 5 hours p.i., the infected cells were stringently washed to remove free virus as well as cell-surface-associated virus, and intracellular viral RNA was quantified. As shown in Figure 2D, viral RNA levels in $\mathrm{Peli1}^{-/}$macrophages were $27 \%$ to $54 \%$ lower than those in WT cells. These results indicated that Peli1 is involved in WNV attachment and entry. To confirm these results, we performed ultrastructural analysis of macrophages from both groups of mice 0 minutes, 5 minutes, and 10 hours after exposure to WNV (Figure 2, E and F). At 0 hours, we detected $16 \mathrm{WNV}$ particles, ranging from 41 to $46 \mathrm{~nm}$ in diameter, in association with the plasma membrane in WT macrophages, whereas a total of 7 WNV particles were found in association with the plasma membrane of Peli1 $^{--}$macrophages. At 5 minutes p.i., we detected 38 viral particles with a diameter of approximately $42 \mathrm{~nm}$ in small, uncoated vesicles of the WT macrophages. However, only $11 \mathrm{WNV}$ particles of the same size were identified in Peli1 ${ }^{-/-}$macrophages. By 10 hours, there were 55 WNV viral particles with a diameter of $42 \mathrm{~nm}$ inside the double-membrane vesicles of WT macrophages. Interestingly, we found 18 viral particles of much smaller size $(\sim 25$ $\mathrm{nm}$ ) inside the double-membrane vesicles of $\mathrm{Peli1}^{-/-}$macrophages (Figure 2, E and F). On day 4 p.i., viral particles detected in the supernatants of WT macrophages ranged in diameter from $41 \mathrm{~nm}$ to $47 \mathrm{~nm}$, which are dimensions similar to those previously reported (28), whereas virions detected in the supernatant of Peli1 ${ }^{-/-}$ macrophages showed substantial variations in size, ranging from 23.5 to $39.5 \mathrm{~nm}$ (Figure 2G). These observations suggest that Peli1 not only plays a role in initial cell attachment and entry, but also in other aspects of the WNV life cycle within the host cells. We next determined the infectivity of WNV that was passaged in WT or Peli1 ${ }^{-/-}$macrophages. WNV passaged in $\mathrm{Peli1}^{-/-}$macrophages had a lower replication rate in WT macrophages compared with WNV passaged in WT macrophages (Figure $2 \mathrm{H}$ ). Furthermore, we challenged WT and Peli1 ${ }^{-/-}$mice with 100 FFU WNV passaged in WT or Peli1 $^{-/-}$macrophages. WT mice infected with WNV passaged in Peli1 ${ }^{-1}$ macrophages showed an increased survival rate $(28.5 \%$, Figure 2I) compared with that of WT mice infected with WNV passaged in WT cells $(0 \%)$. Peli1 ${ }^{-1-}$ mice infected with WNV passaged either in WT macrophages $(43 \%)$ or Peli1 ${ }^{-/}$cells (56\%) also showed a similar resistance to lethal WNV infection.

We next determined whether Peli1 mediates immune responses during WNV infection in macrophages and DCs. Following WNV infection, we found that Peli1 expression was upregulated in macrophages by day 4 . In contrast, the other 2 Pellino family members, including Peli2 and Peli3, remained at low levels (Figure 3A). Compared with WT cells, Peli1 ${ }^{-/}$macrophages had reduced Ifna and Ifn $b$ RNA levels on days 1 and 4 after WNV infection (Figure 3, B and C). Production of inflammatory cytokines, including IL-6 (Il6), TNF- $\alpha$ (Tnfa), and IL-12 (Il12), was also impaired in Peli1 $^{-/}$macrophages by day 4 (Figure 3, D-F). WNV infection in $\mathrm{Peli1}^{-/-}$DCs also resulted in diminished levels of Ifnb, Il1b, and Il12 compared with WT DCs (Supplemental Figure 3C). PRRs, including TLR3 and TLR7, and retinoid acid-inducible gene I-like receptors (RLR)s, such as retinoid acid-inducible gene I (RIG-I) and melanoma differentiation antigen 5 (MDA-5), are involved in WNV recognition and trigger the signaling cascade leading to the production of type 1 IFNs and proinflammatory cytokines (25, 29-31). To understand the role of Peli1 in PRR-mediated signaling pathways, we treated WT and Peli1 ${ }^{-1}$ macrophages with TLR agonists (Poly I:C for TLR3, CL097 for TLR7, and Poly I:C LyoVec 
A
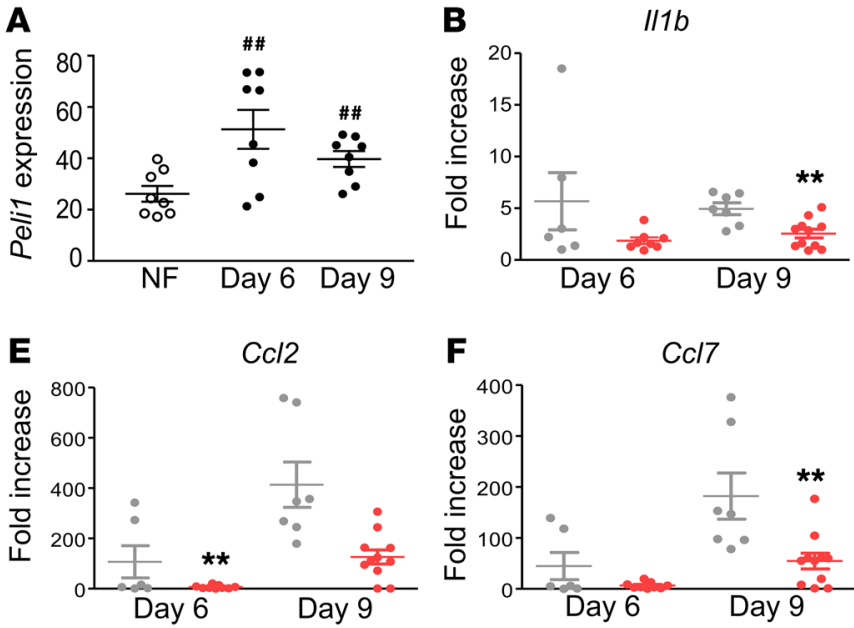
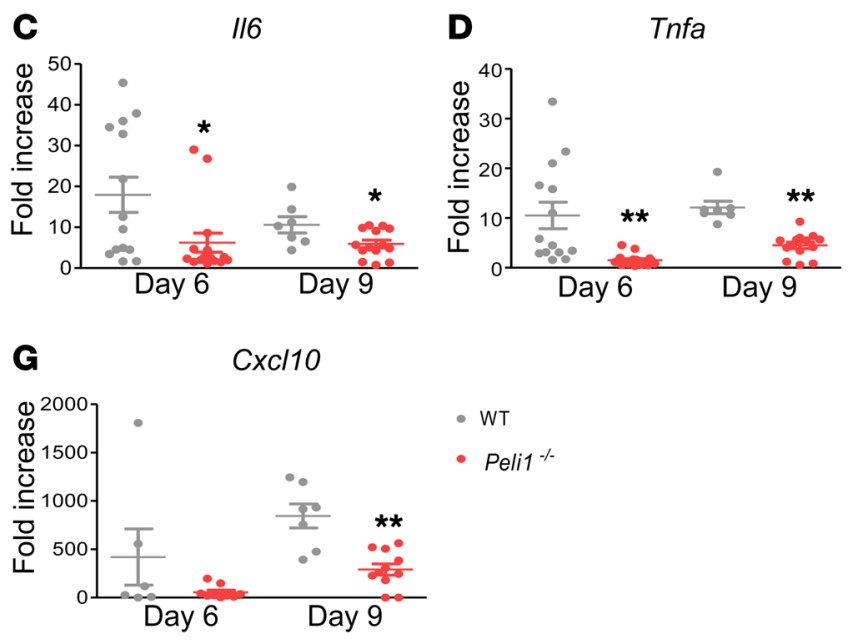
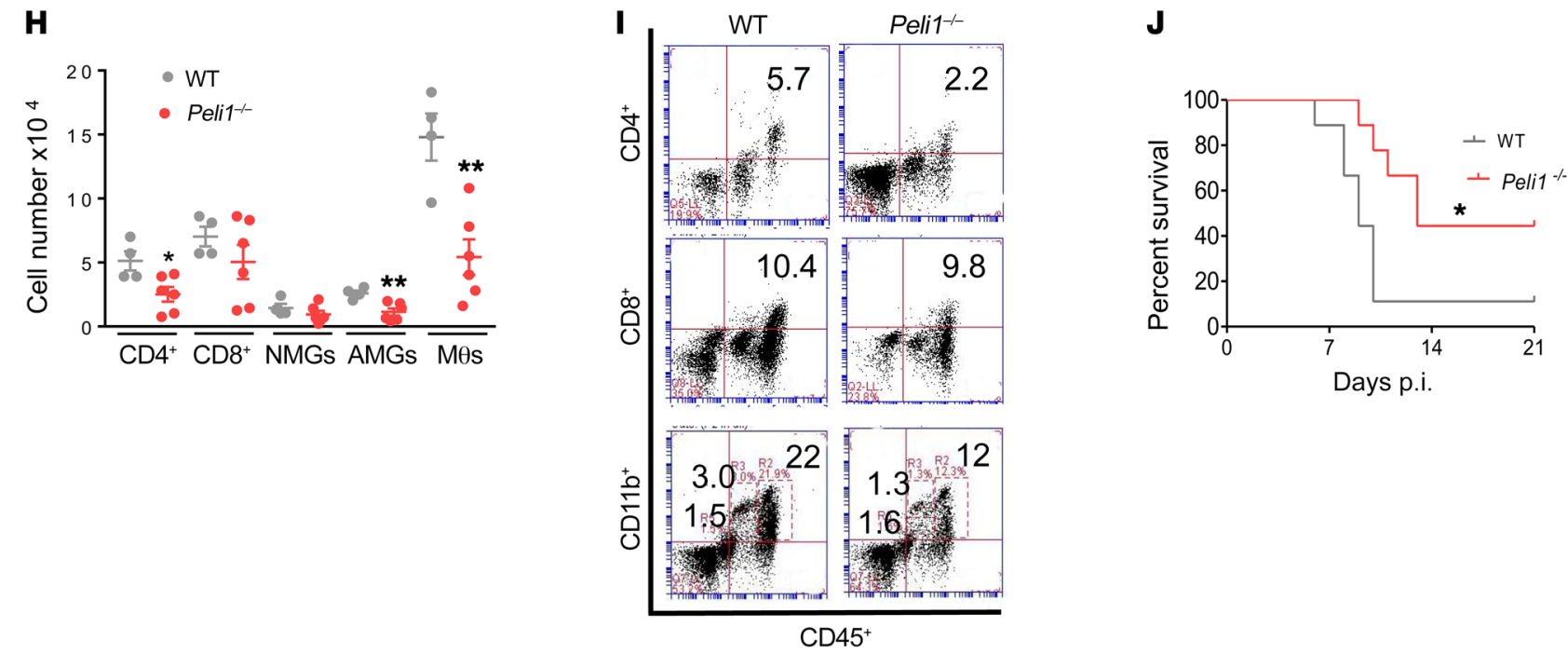

Figure 4. Peli1 mediates neuroinflammation in the CNS after WNV infection. (A) RNA levels of Peli1 in the brains of WT mice following WNV infection were determined by qPCR assay. Data are presented as the mean \pm SEM of samples pooled from 2 to 3 independent experiments $(n=8)$. \#\# $P<0.01$ compared with the noninfected group (unpaired, 2-tailed Student's $t$ test). (B-G) RNA levels of cytokines and chemokines in the brain at the indicated time points were determined by qPCR assay. Data are presented as the mean \pm SEM of 6 to 10 samples pooled from 2 independent experiments. (H-J) Brain leukocyte infiltration following WNV infection. (H) The number of brain leukocytes is presented as the mean \pm SEM of 9 to 10 mice from 2 independent experiments, including naive microglial cells (NMGs), activated microglial cells (AMGs), macrophages (M $\theta s)$, and CD4+ and CD8 ${ }^{+} T$ cells on day 9 p.i. (analyzed by flow cytometry). (I) Representative flow plot. (B-H) ${ }^{*} P<0.05$ and ${ }^{*} P<0.01$ compared with the WT group (unpaired, 2 -tailed Student's $t$ test). (J) Survival of WT and Peli1 ${ }^{-/}$mice after i.c. injection with WNV 385-99 ( $n=9$ per group). ${ }^{*} P<0.05$ compared with the WT group (log-rank test).

for RLRs). We found that both Poly I:C and Poly I:C/LyoVec triggered lower Ifna, Ifnb, Il6, and Il12 expression levels in Peli1 ${ }^{-/}$macrophages. In contrast, stimulation with the TLR7 agonist induced similar antiviral cytokine responses in both groups of macrophages (Supplemental Figure 3, D-G). Peli1 is known to be dispensable for MyD88-dependent TLRs but is required for TRIF-dependent TLR signaling in macrophages and DCs (1). Consistent with the previous findings, we demonstrate that Peli1 positively regulates TLR3- and RLR-mediated, but not TLR7-mediated, innate cytokine responses in macrophages. Finally, to understand the effects of defective viral replication on antiviral immunity, we infected WT macrophages with the same dose of WNV passaged once in WT or Peli1 $1 /$ macrophages. Interestingly, WNV passaged in Peli1 ${ }^{-1-}$ cells triggered diminished Ifnb RNA and IL-6 protein levels in WT macrophages compared with WNV passaged in WT macrophages.
The levels of reduction induced by WNV passaged in Peli1 ${ }^{--}$cells were similar to cytokine levels in Peli1 ${ }^{-/}$cells induced by WT virus (Figure 3G and Supplemental Figure 3H). Collectively, these data show that Peli1 positively mediates antiviral cytokine responses mainly by a direct involvement in the WNV replication life cycle in macrophages and DCs.

Peli1 is predominantly involved in CNS inflammation during $W N V$ infection. Peli1 was previously reported to be highly expressed by neural tissues (2). Here, we noted that Peli1 expression was induced in the brain on days 6 and 9 p.i. (Figure 4A). The mRNA levels of inflammatory cytokines including $I l 1 b, I l 6$, and Tnfa and chemokines such as $\mathrm{Ccl} 2, \mathrm{Ccl} 7$, and $\mathrm{Cxcl10}$ were decreased in Peli1 ${ }^{-/}$mice (Figure 4, B-G). Ifnb, but not Ifna, RNA levels were also reduced in Peli1 ${ }^{-/}$mouse brains (Supplemental Figure 4, A and B). To study the brain leukocyte phenotype, we 
A

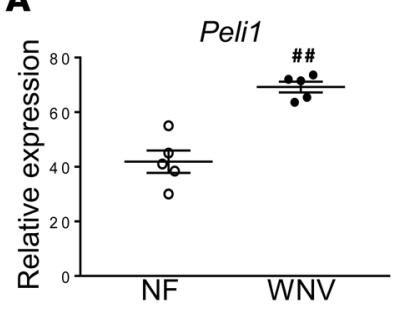

E

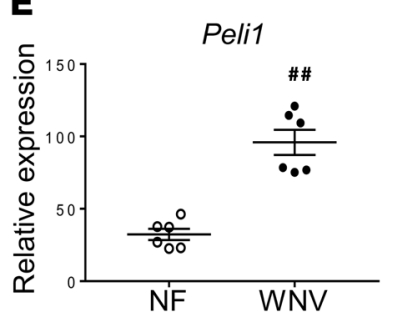

B

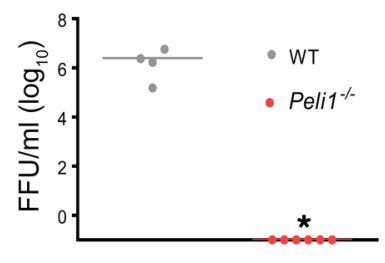

$\mathbf{F}$

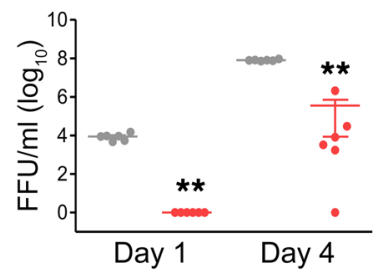

C

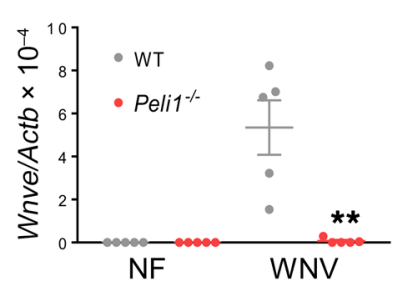

G

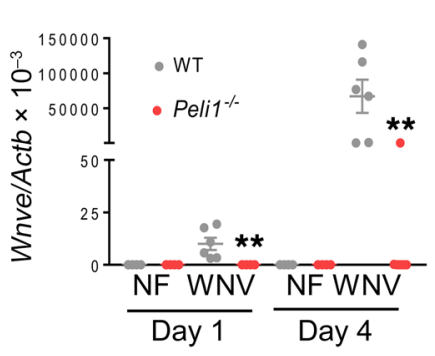

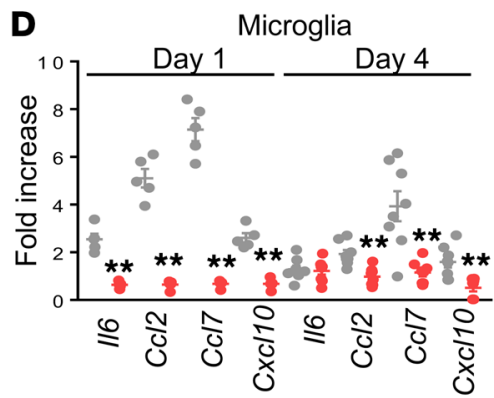

H

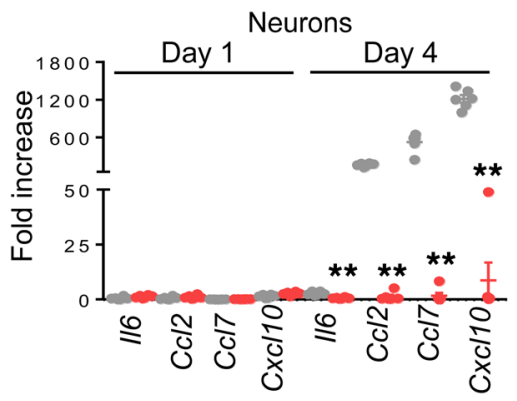

Figure 5. WNV infection and induction of immune responses in microglia and neurons. (A) RNA levels of Peli1 in WT microglia on day 4 p.i. were determined by qPCR. Data are presented as the mean \pm SEM and are representative of 2 independent experiments $(n=5)$. \#\#P<0.01 compared with the noninfected group (unpaired, 2-tailed Student's $t$ test). (B-D) Primary microglia were infected with WNV $385-99$ and harvested at the indicated time points. Data are presented as the mean \pm SEM of 4 to 8 pooled samples from 2 independent experiments. (B and C) Viral load was measured on day 4 . (D) Cytokine RNA levels were measured by qPCR at the indicated time points. (E-H) Primary mouse neurons were infected with WNV $385-99$ and harvested at the indicated time points. Data are presented as the mean \pm SEM ( $n=6$ per group). (E) RNA levels of Peli1 in WT neurons on day 4 p.i. \#\# $P<0.01$ compared with the noninfected group (unpaired, 2-tailed Student's $t$ test). (F and $\mathbf{G}$ ) Viral load was measured at the indicated time points by qPCR (F) and FFA (G. (H) $/ / 6, C c / 2, C c / 7$, and $C x c / 10$ RNA levels were measured by $q P C R$ at the indicated time points. (B-D and $\mathbf{F}-\mathbf{H}){ }^{*} P<0.05$ and ${ }^{* *} P<0.01$ compared with the WT group (unpaired, 2-tailed Student's $t$ test).

performed flow cytometric analysis of these cells isolated on day 9 and found that the number and percentage of infiltrating $\mathrm{CD} 4^{+}$ $\mathrm{T}$ cells, activated microglial cells $\left(\mathrm{CD} 11 \mathrm{~b}^{\mathrm{hi}} \mathrm{CD} 45^{\mathrm{lo}}\right)$, and macrophages $\left(\mathrm{CD} 11 \mathrm{~b}^{\mathrm{hi}} \mathrm{CD} 45^{\text {hi }}\right)$ were decreased by up to $60 \%$ in $\mathrm{Peli1}^{-1-}$ mice (Figure 4, H and I, and Supplemental Figure 4C). To exclude the effects of Peli1 on WNV infection in peripheral tissues, we inoculated mice from both groups with WNV intracranially (i.c.) (Figure 4J). Peli1 ${ }^{--}$mice showed resistance that was similar to that seen with systemic WNV infection (44\% versus $11 \%$, Peli1 ${ }^{-/}$versus WT). Collectively, these results indicate that Peli1 is involved in WNV encephalitis predominantly by mediating CNS infection and inducing neuroinflammation.

Peli1 promotes microglia activation via facilitation of $W N V$ replication in neurons and microglia in mice and humans. Microglial cells are involved in CNS neuroinflammation $(24,32)$ and are permissive to WNV infection (Supplemental Figure 5A). Peli1 is highly expressed on microglial cells (2) and was enhanced following WNV infection, however, Peli2 and Peli3 remained at low levels (Figure 5A and Supplemental Figure 5B). WNV triggered higher mRNA levels of inflammatory cytokines (Tnfa and Il12) and chemokines ( $\mathrm{Ccl} 2, \mathrm{Ccl} 4$, and $\mathrm{Cxcl10}$ ) in microglial cells (Supplemental Figure 5C). Interestingly, we observed that WNV infection was nearly abolished in both Peli1 ${ }^{-/}$primary microglial cells (Figure 5, B and C) and Peli1-depleted microglial cells (Supplemental Figure 5D), which was accompanied by diminished levels of inflammatory cytokines and chemokines (Figure 5D and Supplemental Figure 5E). Neurons are most permissive to WNV infection in the CNS $(18,33)$. WNV infection upregulated Peli1 expression on neurons by up to $200 \%$ (Figure $5 \mathrm{E}$ ). WNV replication was also significantly reduced in $\mathrm{Peli1}^{-/}$neurons compared with that seen in WT neurons (Figure 5, F and G). We found that mRNA levels of chemokines ( $\mathrm{Ccl} 2, \mathrm{Ccl}$, and $\mathrm{Cxcl1O})$ and inflammatory cytokines (Il6 and Ifnb) were all diminished in WNV-infected Peli1 ${ }^{-/}$neurons by day 4 (Figure $5 \mathrm{H}$ and Supplemental Figure 5F). Western blot analysis showed that phosphorylation levels of p38MAPK and p65 were both reduced in WNV-infected Peli1 ${ }^{--}$neurons on day 3 compared with levels in WT controls (Supplemental Figure 5, G and $\mathrm{H}$ ), which suggests a role of Peli1 in the positive regulation of $\mathrm{NF}-\mathrm{kB}$ and $\mathrm{p} 38 \mathrm{MAPK}$ activation.

Immunostaining of postmortem hippocampal tissues from patients who died of acute WNV encephalitis showed membrane Peli1 on WNV-positive neurons and adjacent inflammatory cells, but not in the same region of age-matched control patients (Figure 6A). Peli1 expression was also upregulated following in vitro WNV infection in SHSY-5Y-differentiated neurons and HMC3 cells (a human microglial cell line) (Figure 6, B and C) and WNV-infected PBMCs (Supplemental Figure 6A). Nevertheless, its expression was not changed in WNV-infected neural stem cell-derived neurons or in THP1-derived macrophages (Supplemental Figure 6A). Knockdown (KD) of Peli1 expression in human microglial cells or SHSY-5Y-differentiated neurons decreased viral loads by $20 \%$ to $35 \%$ by day 4 p.i. (Figure 6, D and E, and Supplemental Figure 6B). Peli1 deficiency also decreased IL-6, CCL2, and CCL5 production in human microglial cells (Figure 6, F-H). WNV infection did not 

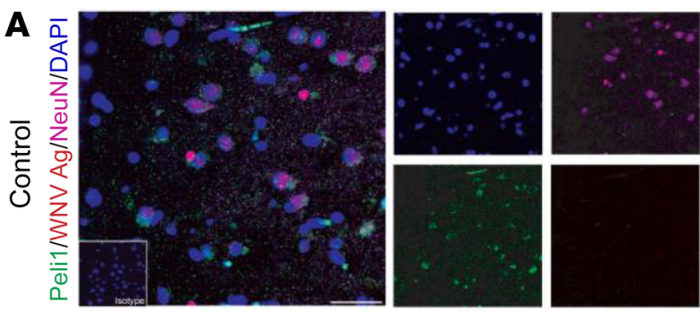

\section{B}
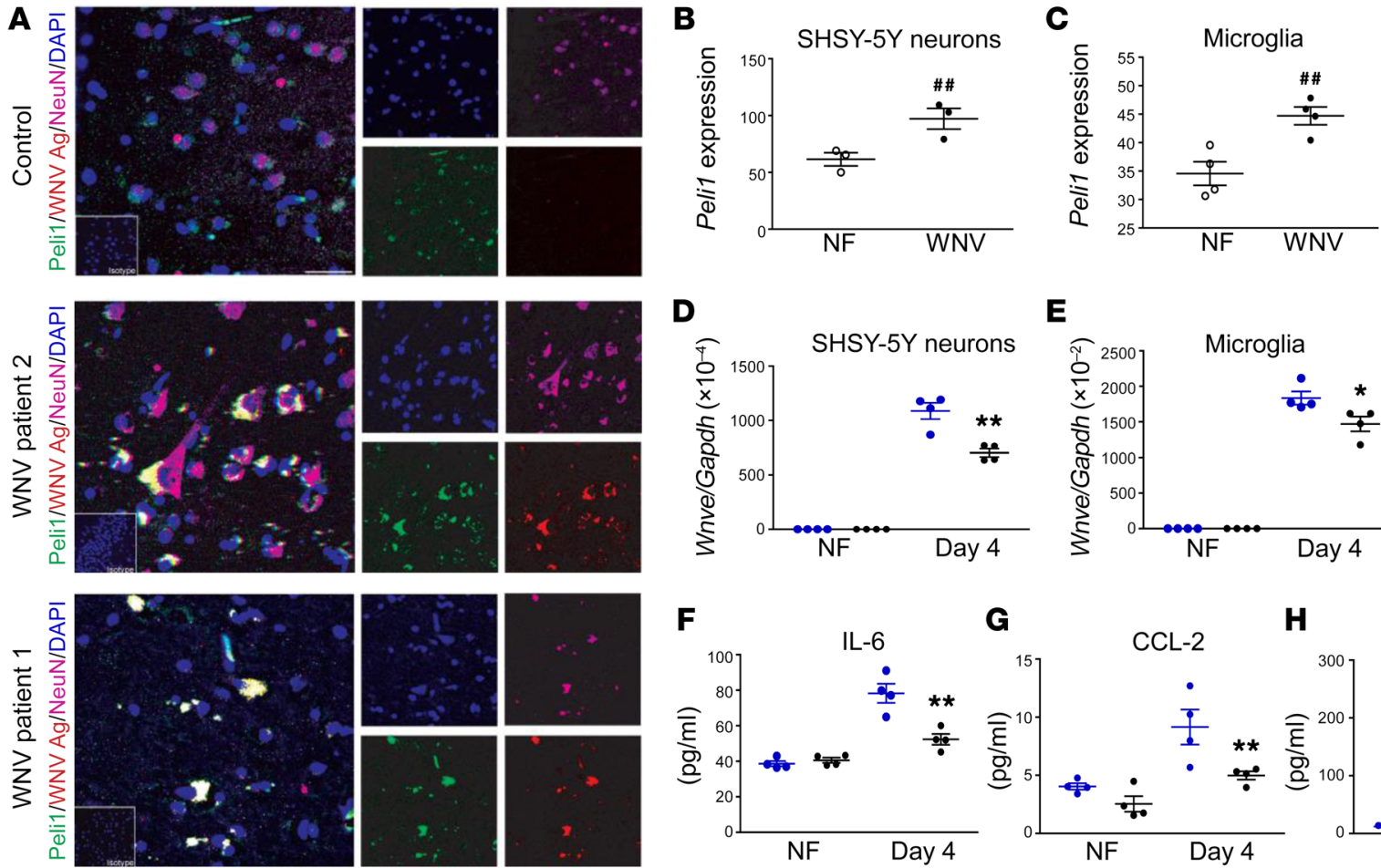

D

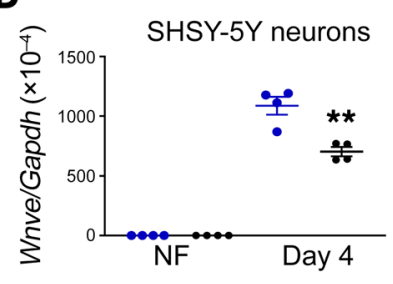

E

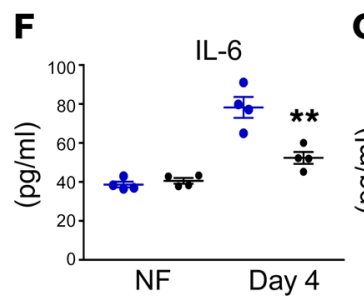

G

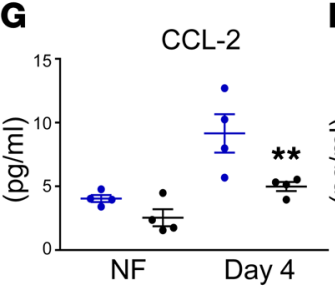

Microglia

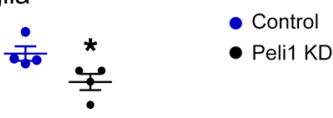

Figure 6. Peli1 expression and its role in human cells during WNV infection. (A) Immunodetection of Peli1 (green), WNV antigen (WNV Ag) (red), and NeuN (purple), in postmortem hippocampal tissues from 1 control and 2 WNV-infected patients. Nuclei were counterstained with DAPI (blue). Scale bar: 8 $\mu \mathrm{m}$. Insets are images of sections stained with isotype control antibodies or serum (original magnification, $\times 63$ ). (B and C) RNA levels of Peli1 in SH-SY5derived neurons and human microglia on days 1 and 4 p.i. were determined by qPCR. Data are presented as the mean \pm SEM and are representative of 2 similar experiments $(n=3-4)$. ${ }^{\# \#} P<0.01$ compared with the noninfected group (unpaired, 2-tailed Student's $t$ test). (D-H) SHSY-5Y-derived neurons (D) and human microglial cells (E-H) were treated with control or Peli1 siRNA (Peli1 KD), infected with WNV $385-99$ at 48 hours, and harvested at the indicated time points. ( $\mathbf{D}$ and $\mathbf{E}$ ) Viral load was measured on day 4 by qPCR. (F-H) IL-6, CCL-2, and CCL-5 production in microglial cells was measured on day 4 by BioPlex assay. Data are presented as the mean \pm SEM and are representative of 2 similar experiments $(n=4)$. (D-H) ${ }^{*} P<0.05$ and ${ }^{* *} P<0.01$ compared with the control group (unpaired, 2-tailed Student's $t$ test).

induce inflammatory cytokine or chemokine production in human neurons (data not shown). We noted that Peli1 KD (36\% reduction, Supplemental Figure 6C) in human fetal cortical neural stem cellderived (hNSC-derived) neurons led to a $30 \%$ decrease on Ifnb levels on day 4 p.i. compared with levels in control siRNA-treated neurons (Supplemental Figure 6D). These data suggest that Peli1 is involved in WNV replication in neural cells and induction of inflammatory cytokines and chemokines in human microglial cells.

To determine whether defective replication contributes to attenuated inflammatory responses in the CNS, we infected WT microglial cells with WNV passaged in WT or Peli1 ${ }^{-/-}$neurons or macrophages. WT microglia infected with WNV grown in Peli1 ${ }^{-1-}$ cells had lower viral titers (Figure 7, A and B) and reduced mRNA levels of Il6, Ccl2, and Ccl7, but not of Tnfa or Cxcl1O (Figure 7, C-E), compared with cells infected with WNV grown in WT cells. It is known that microglia respond to viral infection via activation of p38MAPK (24). Microglia infected with WNV passaged in $\mathrm{Peli1}^{-/}$cells also showed lower phosphorylation levels of $\mathrm{p} 38 \mathrm{MAPK}$ than did cells infected with WNV passaged in WT neurons (Figure 7F). Smaducin-6, a membrane-tethered, palmitic acid-conjugated peptide composed of amino acids 422-441 of Smad6, was reported to interact with Peli1 and disrupt the formation of IRAK1, RIP-1, and IKK, but not MAPK-mediated signaling complexes (34). Smaducin-6 treatment did not block WNV infection or induction of inflammatory cytokine/chemokine production in microglial cells or macrophages, though it decreased the levels of type 1 IFNs in macrophages (Figure 7, G and $\mathrm{H}$, and Supplemental Figure 7). Overall, our results suggest that Peli1 is required for WNV replication in neural cells, which promotes p38MAPK activation in microglia and induction of inflammatory immune responses in the CNS.

\section{Discussion}

Peli1 has been reported to be an important mediator in the activation of NF- $\mathrm{BB}$ or $\mathrm{p} 38 \mathrm{MAPK}$ in TLR-dependent signaling pathways (2). In this study, we have provided evidence that Peli1 facilitates WNV replication and mediates innate immunity in the periphery and CNS (Figure 8). In particular, on the basis of several facts, we conclude that Peli1 is predominantly involved in CNS neuroinflammation during WNV infection. First, although Peli1 promotes WNV replication in myeloid cells (macrophages and DCs), it also positively regulates antiviral innate immune responses in these cells, which compromise its overall pathogenic effects in the periphery. Second, compared with myeloid cells, Peli1 expression is highly enriched in CNS-resident cells and is even upregulated following WNV infection. Third, Peli1 facilitates WNV replica- 

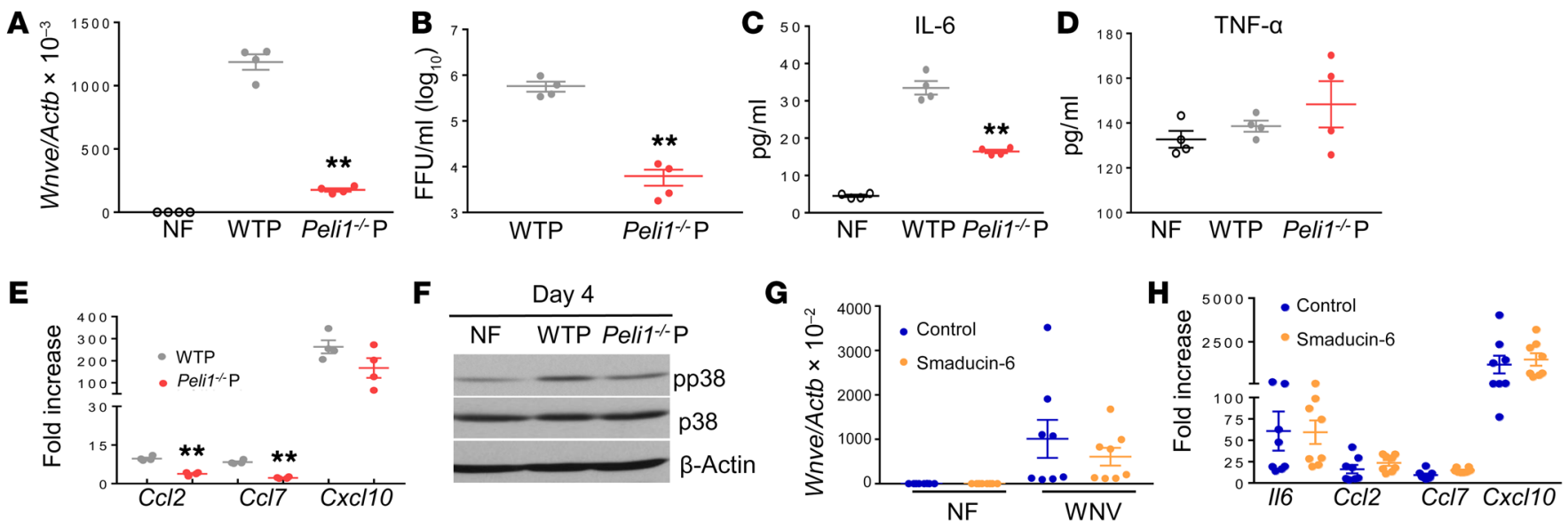

Figure 7. Peli1 promotes p38MAPK activation in microglia via facilitation of WNV replication. (A-F) BV2 cells were infected at a MOI of 0.02 with viruses passaged in WT (WTP) and Peli $7^{-1-}\left(\right.$ Peli ${ }^{-1-}$ P) macrophages or neurons. (A and B) On day 4 p.i., the viral load was measured by qPCR (A) or FFA (B). (C-E) IL- 6 and TNF- $\alpha$ production and $C \mathrm{C} / 2, C \mathrm{Cl}$, and $C x \mathrm{C} / 10$ RNA levels were measured on day 4 by Bio-Plex or $\mathrm{PPCR}$, respectively. Data are presented as the mean \pm SEM and are representative of 2 similar experiments $(n=4)$. (A-E) ${ }^{* *} P<0.01$ compared with the WT group (unpaired, 2-tailed Student's $t$ test). (F) Western blot assay for p38MAPK activation. One representative sample of two per group is shown. (G and $\mathbf{H})$ BV2 cells were infected at a MOI of 0.02 with WNV 385-99 and treated with Smaducin-6 or control peptides 1 hour p.i. (C) Viral load was measured by qPCR on day 4 p.i. (H) Cytokine and chemokine levels were measured on day 4 by qPCR. Data are presented as the fold increase compared with mock-infected cells (mean \pm SEM) and represent 8 samples pooled from 2 independent experiments.

tion in microglia and neurons, especially in the latter, which are the major cells infected during in vivo challenge. It also positively mediates NF- $\mathrm{KB}$ and/or p38MAPK activation in these cells and boosts a robust production of inflammatory cytokines and chemokines, which attracts more inflammatory cells infiltrated from the periphery and ultimately contributes to lethal WNV encephalitis. Thus, Peli1 synergistically promotes virus dissemination and inflammation in the CNS. Last, Peli1 ${ }^{-/-}$mice had similar levels of resistance compared with WT mice following systemic and direct intracranial WNV infection. This further indicates that Peli1 promotes WNV-induced pathology primarily in the CNS.

WNV-induced CNS disease is partially caused by bystander damage from both the immune response induced in the CNS-residential cells $(17,18)$ and by infiltrating inflammatory cells (19-23). As shown in Figure $4 \mathrm{H}$, Peli1 $^{-/-}$mouse brains had a significantly decreased number of infiltrating $\mathrm{CD}^{+} \mathrm{T}$ cells $(P<0.05)$, activated microglia $(P<0.01)$, and macrophages $(P<0.01)$. Both CCL2 and CCL7 are involved in monocytosis and monocyte accumulation in the brain (35), whereas CXCL10 helps recruit antigen-specific $\mathrm{T}$ cells (36). Microglial cells are the major producers of inflammatory cytokines and chemokines in the CNS following WNV infection $(25,37)$. In vitro WNV infection in $\mathrm{Peli1}^{-/-}$or Peli1-depleted mouse and human microglia resulted in a lower viral load and induced impaired production of IL-6, CCL2, CCL7, and CXCL10. Neurons are the primary targets during in vivo WNV replication in the CNS $(18,38)$. WNV passaged in Peli1 ${ }^{-/}$neurons induced similarly lower levels of p38MAPK phosphorylation in WT microglia, and this was accompanied by impaired production of the chemokines CCL2 and CCL7, but not CXCL10, in these cells. Collectively, our results suggest that Peli1 mediates proinflammatory cytokine and chemokine production predominantly via facilitation of WNV replication in neural cells (microglia and neurons), which ultimately leads to macrophage/monocyte and $\mathrm{T}$ cell infiltration into the CNS.
In line with findings of a previous report (37), we did not note a significant induction of inflammatory cytokine responses in human neurons following WNV infection. Interestingly, WNV induced higher Peli1 expression on mouse neurons than on microglia. WNV-infected Peli1 ${ }^{-/-}$mouse neurons had reduced levels of NF- $\mathrm{BB}$ and $\mathrm{p} 38 \mathrm{MAPK}$ activation, accompanied by impaired production of inflammatory cytokines and chemokines. We noted that noninfected Peli1 ${ }^{--}$mouse neurons also had lower levels p38MAPK activation, despite normal basal levels of cytokine production compared with WT controls (data not shown). Thus, it is likely that Peli1 positively regulates inflammatory cytokine and chemokine responses in WNV-infected mouse neurons via NF- $\mathrm{BB}$ activation. Peli1 is known to activate NF- $\kappa \mathrm{B}$ signaling via interaction with RIPK1, another RIPK family member, which was recently shown to mediate neuronal chemokine induction and recruit $\mathrm{T}$ lymphocytes and inflammatory myeloid cells into the CNS (39). Whether Peli1 interacts with RIPK3 and regulates inflammatory responses remains to be investigated.

Smad6 and Smad7 are critical mediators for effective TGF- $\beta 1-$ mediated suppression of IL-1R/TLR signaling via their simultaneous binding to discrete regions of Peli1 (40). Smaducin-6, which is composed of amino acids 422-441 of Smad6, has been reported to disrupt the formation of IRAK1-, RIP1-, and IKKe-mediated TRIF signaling complexes, but not the phosphorylation of $\mathrm{p} 38 \mathrm{MAPK}$ in macrophages (34). We found that Smaducin-6 did not inhibit WNV replication in microglia or macrophages. Further, Smaducin- 6 did not block the production of inflammatory cytokines or chemokines in WNVinfected microglia or macrophages. These results support a role of Peli1 in the induction of inflammatory responses in both cell types during WNV infection via the promotion of p38MAPK activation.

Consistent with the findings in myeloid and CNS-resident cells, the production of type I IFNs, inflammatory cytokines, and chemokines in the blood and CNS tissues were all impaired 


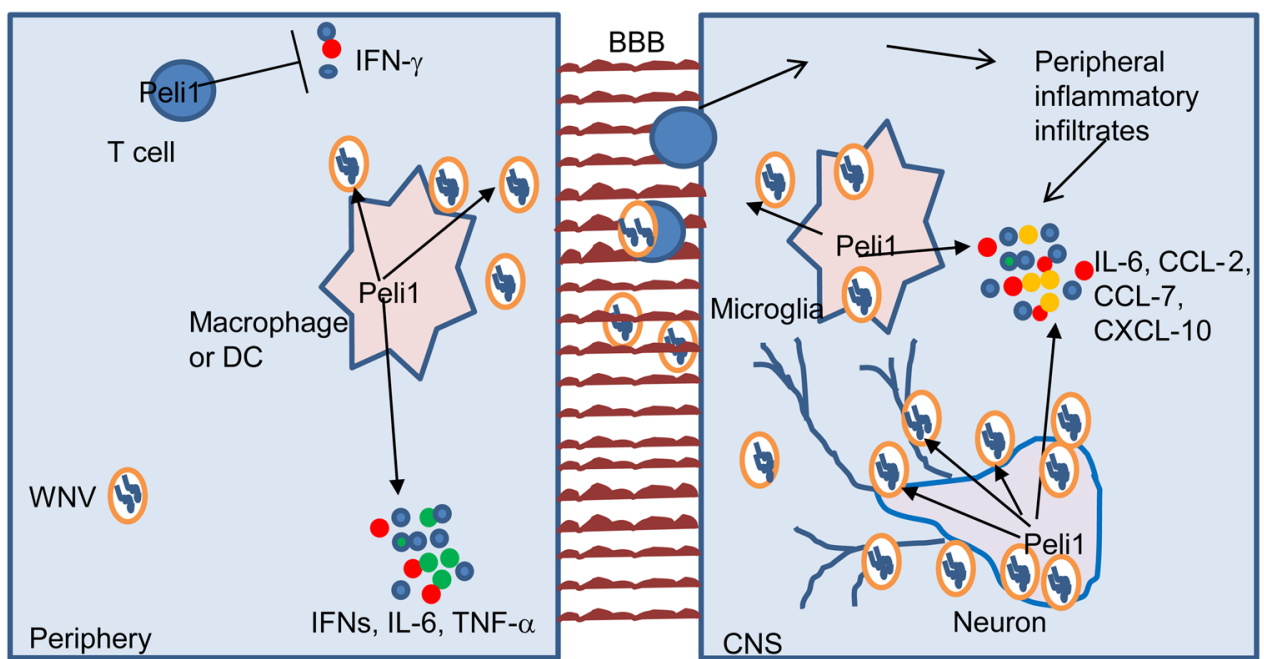

Figure 8. Proposed model illustrating how Peli1 promotes virus replication and neuroinflammation during WNV infection. In the periphery, Peli1 promotes WNV replication in macrophages and DCs and positively regulates antiviral innate immune responses in these cells. Peli1 also negatively mediates IFN- $\gamma$ production in T cells. Once the virus crosses the BBB and enters the CNS, Peli1 facilitates WNV replication in microglia and neurons and further boosts a robust inflammatory cytokine and chemokine production in these cells, which attracts more inflammatory cells infiltrated from the periphery and ultimately contributes to lethal WNV encephalitis. in WNV-infected Peli1 ${ }^{-/}$mice. Our results suggest a role of Peli1 as a positive regulator of innate immunity. There are conflicting reports on the role of Peli1 in the induction of type I IFN responses during viral infection. For example, Xiao et al. found that Peli1 negatively regulates IFN signaling in microglia and macrophages during vascular stomatitis viral infection in the CNS (41). Another group has shown that Peli1 interacts with DEAF1 and positively regulates IFN- $\beta$ production following Sendai virus infection (42). While our results are in line with the latter finding, the main difference between ours and the previous findings is that Peli1 positively mediates immune induction predominantly via the facilitation of WNV entry and replication.

The WNV life cycle includes attachment/entry, translation, RNA replication, and egress of viral particles. Although the virus relies heavily on host proteins during its life cycle $(43,44)$, our understanding of the molecular interactions of viruses and mammalian host cells and their effects on viral pathogenesis is limited. Previous RNA interference screening of human genes responsible for interaction with WNV proteins identified the Ub ligase CBLL1 as being critical for WNV internalization during in vitro infection (45). Several host factors, including AXL and TIM1, have been shown to be involved in flavivirus replication from in vitro cell culture studies. However, their roles remain to be confirmed in vivo (46-48). In this study, by using both in vivo and in vitro models, we identified Peli1 as a host factor required for WNV initial cell attachment and entry, a process that further promotes TLR-mediated inflammatory responses in mouse and human neural cells. Interestingly, the size of the virions generated after passaging WT WNV once in Peli1 $^{--}$cells was markedly reduced. We noted no genetic changes in the passaged viruses (data not shown). However, the reduction of virion size is likely due to an altered protein composition or changes in the ratio of viral proteins in the virions during virus assembly (49). In particular, the smaller viral particles produced in Peli1 $^{-/-}$cells are consistent with the size of recombinant subviral particles assembled in the endoplasmic reticulum during flavivirus infection in mammalian cells reported previously (50). The subviral particles consist of the premembrane-E (prM-E) structural proteins, retain functional properties, and are transported from the endoplasmic reticulum through the secretory pathways to undergo cleavage maturation by the cellular protease furin. Mutation of the furin recognition site of the prM of the tick-borne encephalitis virus resulted in secretion of the smaller subviral particles. Altogether, our data suggest that Peli1 is involved in WNV attachment, entry, and assembly. The smaller virions generated from $\mathrm{Peli1}^{-/-}$cells had reduced replication rates in WT cells and triggered attenuated inflammatory responses in these cells, all of which contributed to a higher resistance in WT mice. These results further support our hypothesis that Peli1 mediates inflammatory responses and promotes WNV encephalitis by facilitating virus replication. Consistent with our findings in the mouse model, we also demonstrate that Peli1 expression is associated with WNV infection and inflammatory cell activation in postmortem hippocampal tissues from patients who died of acute WNV encephalitis. Results from this study provide us with a better understanding of the mechanisms by which WNV induces lethal encephalitis. We believe our findings will ultimately help to identify new therapeutic targets such as Peli1 and lead to the development of virus replication inhibitors to prevent and treat WNV-induced encephalitis. Last, Peli1 is expressed on many cell types and is highly enriched in CNS tissues. Future studies will be needed to determine the role of Peli1 in other virus models, in particular in neurotropic flavivirus models.

\section{Methods}

Further information can be found in the Supplemental Methods.

Mice. Five- to eight-week-old C57BL/6 (B6) mice were purchased from The Jackson Laboratory. Peli1 ${ }^{-/-}$mice (on a B6 background) (2, 51) were bred at UTMB. Both female and male mice were used in this study and were age and sex matched. Mice were inoculated i.p. with 100 FFU WNV 385-99 $(52,53)$ or WNV 385-99 passaged once in WT and $\mathrm{Peli1}^{-/-}$macrophages. In some experiments, mice were challenged intracranially (i.c.) with 5 FFU WNV 385-99.

Cells. BM-derived DCs, macrophages, and primary microglia cultures were isolated as described previously $(2,54)$. Neurons were generated as described previously (55), with slight modifications. After the dissection and dissociation of cortices of E18.5 mouse embryos, cells were enriched using a Mouse Neuron Isolation Kit (Miltenyi Biotec) and cultured for 5 days in neurobasal medium containing B-27 supplement (Invitrogen, Thermo Fisher Scientific). BV2 cells were provided 
by A. Cardona (University of Texas San Antonio, San Antonio, Texas, USA). BM-DCs or macrophages, microglia, and BV2 cells were infected with WNV at a MOI of 0.1 or 0.02 , and neurons were infected at a MOI of 0.003. SH-SY5Y cells were cultured in F12K medium and Eagle's minimal essential media (EMEM) (Invitrogen, Thermo Fisher Scientific) and seeded in a 6-well plate for 1 day and then replaced with fresh medium containing $30 \mu \mathrm{M}$ retinoic acid (MilliporeSigma) and 1\% B-27 Supplements (Gibco, Thermo Fisher Scientific, catalog 17504) for 5 days, and the cells differentiated into neurons. Smaducin-6 or Pal-Scram peptides (34) were purchased from MilliporeSigma and used at $100 \mathrm{~nm} 1$ hour after infection. Supernatants and cells were harvested 24 and 96 hours p.i. to measure the viral load and cytokine production. In some experiments, WT and Peli1 $^{-/}$macrophages or neurons were infected with WNV 385-99. Culture supernatants were then harvested on day 4 for virus titration by FFA. Equal titers of viruses from WT and Peli1 ${ }^{-/}$culture were subsequently used for in vivo and in vitro infection studies.

FFA. Vero cells were incubated with sample dilutions for 1 hour. A semisolid overlay containing $0.8 \%$ methylcellulose (MilliporeSigma), 3\% FBS, $1 \%$ penicillin-streptomycin, and 1\% L-glutamine was added. At 48 hours, the semisolid overlay was removed and cells were washed and fixed with 1:1 of an acetone/methanol solution for at least 30 minutes at $-20^{\circ} \mathrm{C}$. Cells were next subjected to immunohistochemical staining with a rabbit WNV polyclonal antibody (World Reference Center for Emerging Viruses and Arboviruses [WRCEVA], T35502) followed by goat anti-rabbit HRP-conjugated IgG (KPL, 474-1516) for 1 hour. Next, cells were incubated with a peroxidase substrate (VECTOR Laboratories) until color developed. The number of foci was used to calculate viral titers.

qPCR. Samples were resuspended in TRIzol (Invitrogen, Thermo Fisher Scientific) for RNA extraction. cDNA was synthesized by using a qScript cDNA Synthesis Kit (Bio-Rad). The sequences of the primer sets for WNV envelope (Wnve), Peli1, Peli2, and Peli3 and cytokine cDNA and PCR reaction conditions were described previously $(2,25$, $36,53,56)$. The assay was performed using the CFX96 Real-time PCR System (Bio-Rad). Gene expression was calculated on the basis of $\mathrm{Ct}$ values using the following formula: $2^{\wedge}$-[Ct(target gene)-Ct(GAPDH or ACTB)] $(53)$.

Viral attachment and entry assays. BM-macrophages were incubated with WNV (MOI of 3 or 10) at $4^{\circ} \mathrm{C}$ for 1 hour, allowing the virus to attach to the cell surface. After 1 hour of incubation, infected cells were washed 3 times with cold PBS to remove unbound virions. Cell-surface-associated viruses were removed by washing with cold alkaline high-salt solution (1 M NaCl and $50 \mathrm{mM}$ sodium bicarbonate, $\mathrm{pH}$ 9.5). After 2 cold PBS washes, cells were harvested and suspended in $3 \mathrm{ml}$ DMEM medium containing $2 \%$ FBS. Total cells were collected by centrifugation at $1,000 \mathrm{~g}$ for 5 minutes. The cell pellets were resuspended in TRIzol for RNA extraction to measure viral titers by qPCR. Some cells were further incubated at $37^{\circ} \mathrm{C}$ to initiate viral entry. At 1.5 and 5 hours p.i., the infected cells were stringently washed to remove free virus as well as cell-surface-associated virus, and intracellular viral RNA levels were quantified by qPCR.

Transmission electron microscopy. Cells were washed with ice-cold PBS and incubated on ice for 15 minutes before exposure to WNV (MOI $=10$ ) for 1 hour. Cells were then rinsed with ice-cold PBS, resuspended in prewarmed media, and incubated at $37^{\circ} \mathrm{C}$. At 0 hours, 5 minutes, and 10 hours p.i., cells were pelleted and fixed for at least 1 hour in a mixture of $2.5 \%$ formaldehyde and $0.1 \%$ glutaraldehyde in 0.05
M cacodylate buffer ( $\mathrm{pH} 7.3$ ), to which $0.01 \%$ picric acid and $0.03 \%$ $\mathrm{CaCl}_{2}$ were added (electron microscopy [EM] fixative). The pellets were washed in $0.1 \mathrm{M}$ cacodylate buffer followed by postfixation in $1 \%$ OsO4 in $0.1 \mathrm{M}$ cacodylate buffer for 1 hour, washing, and then en bloc staining with $2 \%$ aqueous uranyl acetate for 20 minutes at $60^{\circ} \mathrm{C}$. The pellets were next dehydrated in ethanol, processed through propylene oxide, and embedded in Poly/Bed 812 (Polysciences). Sections were cut on a Leica EM UC7 ultramicrotome, stained with lead citrate, and examined with a Philips CM-100 transmission electron microscope (TEM) at $60 \mathrm{kV}$. Images were acquired with a Gatan Orius SC200 digital camera. In some experiments, supernatants of WNV-infected cells were concentrated on day 4 p.i. using 3-kDa spin columns (Sartorius). The concentrated supernatants were centrifuged for 10 minutes at $3,000 \mathrm{~g}$ to remove debris. Next, nickel grids were incubated with clarified supernatants for 10 minutes followed by glutaraldehyde fixation and $2 \%$ uranyl acetate staining. Micrographs were taken using a CM100 TEM (Philips).

Flow cytometry. Brain leukocytes were isolated as described previously (20) and stained with antibodies for cell-surface markers, including CD3 (eBioscience, clone 145-2C11); CD4 (eBioscience, clone GK1.5); CD8 (eBioscience, clone 53-6.7); CD11b (eBioscience, clone M1/70); Gr-1 (eBioscience, clone RB6-8C5); and CD45 (BD Biosciences, clone 30-F11), fixed with 1\% paraformaldehyde in PBS, and examined with a C6 Flow Cytometer (BD Biosciences). Dead cells were excluded on the basis of forward and side light scatter.

Western blot analysis. Protein was dissolved in $1 \times$ SDS loading buffer, separated on SDS-PAGE gels, electroblotted onto nitrocellulose membranes, probed with primary and secondary antibodies, and detected using a Pierce ECL System (Thermo Fisher Scientific). The primary antibodies against phosphorylated p38 (p-p38) (Thr180/Tyr182, no. 9211s), p38 (no. 9212), p-NF-кB RelA (Ser 468, no. 3039), and NF-кB (no. 3034) were from Cell Signaling Technology. Mouse monoclonal anti- $\beta$-actin (MilliporeSigma, clone AC-15) was used as a loading control.

Histology. Mice were transcardially perfused with PBS. Brains and spleens were removed and placed in $4 \%$ paraformaldehyde (PFA) for 3 days at $4^{\circ} \mathrm{C}$, followed by $70 \%$ ethanol before embedment in optimal cutting temperature compound. H\&E staining was performed at the Histopathology Laboratory Core of Baylor College of Medicine (Houston, Texas, USA).

Immunohistochemistry. Paraffin-embedded human hippocampal tissues were obtained from fatal WNV encephalitis cases (provided by Beth Levy, St. Louis University, St. Louis, Missouri, USA) and from control postmortem samples from patients without neurologic diseases. Sections were deparaffinized in xylene, rehydrated in serial dilutions of ethanol, and boiled in citrate buffer ( $\mathrm{pH}$ 6.0) for 30 minutes for antigen retrieval. Next, the sections were incubated for 2 hours at room temperature in blocking solution (10\% serum and 2\% Tween-20 in PBS) to permeabilize and block nonspecific binding. This was followed by incubation with primary antibodies against Peli1 (Santa Cruz Biotechnology, sc-271065, 1:50), WNV antigen (UTMB WRCEVA), T35502, 1:500), and NeuN (MilliporeSigma, clone A60, 1:20) or isotype-matched IgG in PBS supplemented with10\% serum overnight at $4^{\circ} \mathrm{C}$. After washing, the sections were incubated for 1 hour at room temperature with fluorescently conjugated secondary antibodies (Life Technologies, Thermo Fisher Scientific, 1:500). Autofluorescence was darkened using $5 \%$ Sudan black B (diluted in 70\% ethanol) incubated for 10 minutes. After washing, nuclei were counterstained with DAPI, 
and coverslips were applied with ProLong Gold Antifade Mountant (Thermo Fisher Scientific). Immunofluorescence was captured using a Zeiss LSM 510 laser-scanning confocal microscope.

Statistics. Survival curve comparisons were performed using GraphPad Prism software, which uses the log-rank test. Values for viral burden, cytokine production, and antibody and $\mathrm{T}$ cell response experiments are presented as the mean \pm SEM. $P$ values for these experiments were calculated with an unpaired, 2-tailed Student's $t$ test. Statistical significance was accepted at a $P$ value of less than 0.05.

Study approval. All experiments were performed in compliance with and under the approval of the IACUC of UTMB.

\section{Author contributions}

HL, ERW, CS, PW, WZ, SJT, PVA, and TW designed the experiments. ERW, HL, EM, JAS, RW, LLV, SZ, JG, NEB, CC, GX, GL, RT, VLP, and TW performed the experiments. ERW, HL, EM, JAS, SZ, BHP, CC, VLP, WZ, RSK, PVA, and TW analyzed the data. CS, PYS, WZ, and SCS provided key reagents. HL, ERW, JAS, PW, RSK, PVA, and TW wrote the manuscript.

\section{Acknowledgments}

This work was supported in part by the Institute for Human Infections and Immunity at UTMB (to TW), NIH grants R01 AI099123 and R01 AI27744 (to TW), R01NS079166, R01NS095747, and R01DA036165 (to SJT), U19 AI083019 and R01 NS052632 (to RSK), and R01 EY022694 and R01 EY026629 (to WZ). JAS was supported by NIH grant F31 AI124662-01. PVA and VLP were partially supported by NIH grant R24 AI120942. We thank the Texas A\&M Institute for Genomic Medicine for the Peli1 ${ }^{-/-}$mice; A. Cardona from the University of Texas San Antonio; and Partha Sarkar from UTMB for BV2 cells and SHSY-5Y cells; Beth Levy for human postmortem hippocampal tissues; Lan Pang for technique support; and Linsey Yeager for assistance with manuscript preparation.

Address correspondence to: Tian Wang, Departments of Microbiology \& Immunology and Pathology, University of Texas Medical Branch, Keiller 3.118B, 301 University Boulevard, Galveston, Texas, 77555-0609, USA. Phone: 409.772.3146; Email: ti1wang@utmb.edu.
1. Chang M, Jin W, Sun SC. Peli1 facilitates TRIF-dependent Toll-like receptor signaling and proinflammatory cytokine production. Nat Immunol. 2009;10(10):1089-1095.

2. Xiao Y, et al. Peli1 promotes microglia-mediated CNS inflammation by regulating Traf3 degradation. Nat Med. 2013;19(5):595-602.

3. Chang $M$, et al. The ubiquitin ligase Peli1 negatively regulates T cell activation and prevents autoimmunity. Nat Immunol. 2011;12(10):1002-1009.

4. Park HY, et al. Pellino 1 promotes lymphomagenesis by deregulating BCL6 polyubiquitination. J Clin Invest. 2014;124(11):4976-4988.

5. Centers for Disease Control Prevention (CDC). Laboratory-acquired West Nile virus infections United States, 2002. MMWR Morb Mortal Wkly Rep. 2002;51(50):1133-1135.

6. Charatan F. Organ transplants and blood transfusions may transmit West Nile virus. BMJ. 2002;325(7364):566.

7. Petersen LR, Brault AC, Nasci RS. West Nile virus: review of the literature. JAMA. 2013;310(3):308-315.

8. Carson PJ, et al. Long-term clinical and neuropsychological outcomes of West Nile virus infection. Clin Infect Dis. 2006;43(6):723-730.

9. Ou AC, Ratard RC. One-year sequelae in patients with West Nile virus encephalitis and meningitis in Louisiana. J La State Med Soc. 2005;157(1):42-46.

10. Cook RL, et al. Demographic and clinical factors associated with persistent symptoms after West Nile virus infection. Am J Trop Med Hyg. 2010;83(5):1133-1136.

11. Sadek JR, et al. Persistent neuropsychological impairment associated with West Nile virus infection. J Clin Exp Neuropsychol. 2010;32(1):81-87.

12. Nolan MS, Podoll AS, Hause AM, Akers KM, Finkel KW, Murray KO. Prevalence of chronic kidney disease and progression of disease over time among patients enrolled in the Houston West Nile virus cohort. PLoS ONE. 2012;7(7):e40374.

13. Patel H, Sander B, Nelder MP. Long-term sequelae of West Nile virus-related illness: a systematic review. Lancet Infect Dis. 2015;15(8):951-959.

14. Sejvar JJ. Clinical manifestations and outcomes of West Nile virus infection. Viruses. 2014;6(2):606-623.

15. Weatherhead JE, et al. Long-term neurological outcomes in West Nile virus-infected patients: an observational study. Am J Trop Med Hyg. 2015;92(5):1006-1012.

16. Anastasiadou A, Kakoulidis I, Butel D, Kehagia E, Papa A. Follow-up study of Greek patients with West Nile virus neuroinvasive disease. Int J Infect Dis. 2013;17(7):e494-e497.

17. Quick ED, Leser JS, Clarke P, Tyler KL. Activation of intrinsic immune responses and microglial phagocytosis in an ex vivo spinal cord slice culture model of West Nile virus infection. J Virol. 2014;88(22):13005-13014.

18. Shrestha B, Gottlieb D, Diamond MS. Infection and injury of neurons by West Nile encephalitis virus. J Virol. 2003;77(24):13203-13213.

19. Bréhin AC, et al. Dynamics of immune cell recruitment during West Nile encephalitis and identification of a new CD19+B220-BST-2+ leukocyte population. JImmunol. 2008;180(10):6760-6767.

20. Glass WG, Lim JK, Cholera R, Pletnev AG, Gao JL, Murphy PM. Chemokine receptor CCR5 promotes leukocyte trafficking to the brain and survival in West Nile virus infection. J Exp Med. 2005;202(8):1087-1098.

21. Lim JK, Obara CJ, Rivollier A, Pletnev AG, Kelsall $\mathrm{BL}$, Murphy PM. Chemokine receptor Ccr2 is critical for monocyte accumulation and survival in West Nile virus encephalitis. JImmunol. 2011;186(1):471-478.

22. Sitati E, McCandless EE, Klein RS, Diamond MS. CD40-CD40 ligand interactions promote trafficking of $\mathrm{CD}^{+} \mathrm{T}$ cells into the brain and protection against West Nile virus encephalitis. J Virol. 2007;81(18):9801-9811.

23. Sitati EM, Diamond MS. CD4 ${ }^{+}$T-cell responses are required for clearance of West Nile virus from the central nervous system. J Virol. 2006;80(24):12060-12069.
24. Town T, Jeng D, Alexopoulou L, Tan J, Flavell RA. Microglia recognize double-stranded RNA via TLR3. J Immunol. 2006;176(6):3804-3812.

25. Wang T, Town T, Alexopoulou L, Anderson JF, Fikrig E, Flavell RA. Toll-like receptor 3 mediates West Nile virus entry into the brain causing lethal encephalitis. Nat Med. 2004;10(12):1366-1373.

26. Vasek MJ, et al. A complement-microglial axis drives synapse loss during virus-induced memory impairment. Nature. 2016;534(7608):538-543.

27. Lazear HM, et al. IRF-3, IRF-5, and IRF-7 coordinately regulate the type I IFN response in myeloid dendritic cells downstream of MAVS signaling. PLoS Pathog. 2013;9(1):e1003118.

28. Mukhopadhyay S, Kim BS, Chipman PR, Rossmann MG, Kuhn RJ. Structure of West Nile virus. Science. 2003;302(5643):248.

29. Fredericksen BL, Gale M. West Nile virus evades activation of interferon regulatory factor 3 through RIG-I-dependent and -independent pathways without antagonizing host defense signaling. J Virol. 2006;80(6):2913-2923.

30. Daffis S, Samuel MA, Suthar MS, Gale M, Diamond MS. Toll-like receptor 3 has a protective role against West Nile virus infection. J Virol. 2008;82(21):10349-10358.

31. Town T, et al. Toll-like receptor 7 mitigates lethal West Nile encephalitis via interleukin 23-dependent immune cell infiltration and homing. Immunity. 2009;30(2):242-253.

32. Kelley TW, Prayson RA, Ruiz AI, Isada CM, Gordon SM. The neuropathology of West Nile virus meningoencephalitis. A report of two cases and review of the literature. Am J Clin Pathol. 2003;119(5):749-753.

33. Cho H, Proll SC, Szretter KJ, Katze MG, Gale M, Diamond MS. Differential innate immune response programs in neuronal subtypes determine susceptibility to infection in the brain by positive-stranded RNA viruses. Nat Med. 2013;19(4):458-464.

34. Lee YS, et al. Inhibition of lethal inflammatory responses through the targeting of mem- 
brane-associated Toll-like receptor 4 signaling complexes with a Smad6-derived peptide. EMBO MolMed. 2015;7(5):577-592.

35. Bardina SV, et al. Differential roles of chemokines CCL2 and CCL7 in monocytosis and leukocyte migration during West Nile virus infection. JImmunol. 2015;195(9):4306-4318.

36. Klein RS, et al. Neuronal CXCL10 directs CD8 ${ }^{+}$ T-cell recruitment and control of West Nile virus encephalitis. JVirol. 2005;79(17):11457-11466.

37. Cheeran MC, Hu S, Sheng WS, Rashid A, Peterson PK, Lokensgard JR. Differential responses of human brain cells to West Nile virus infection. JNeurovirol. 2005;11(6):512-524.

38. Sampson BA, Ambrosi C, Charlot A, Reiber K, Veress JF, Armbrustmacher V. The pathology of human West Nile virus infection. Hum Pathol. 2000;31(5):527-531.

39. Daniels BP, et al. RIPK3 Restricts viral pathogenesis via cell death-independent neuroinflammation. Cell. 2017;169(2):301-313.e11.

40. Lee YS, et al. Smad7 and Smad6 bind to discrete regions of Pellino-1 via their $\mathrm{MH} 2$ domains to mediate TGF-beta1-induced negative regulation of IL-1R/TLR signaling. Biochem Biophys Res Commun. 2010;393(4):836-843.

41. Xiao Y, Jin J, Zou Q, Hu H, Cheng X, Sun SC. Peli1 negatively regulates type I interferon induction and antiviral immunity in the CNS. Cell Biosci.
2015;5:34.

42. Ordureau A, et al. DEAF1 is a Pellino1-interacting protein required for interferon production by Sendai virus and double-stranded RNA. J Biol Chem. 2013;288(34):24569-24580.

43. Bidet K, Garcia-Blanco MA. Flaviviral RNAs: weapons and targets in the war between virus and host. Biochem J. 2014;462(2):215-230.

44. Brinton MA. Replication cycle and molecular biology of the West Nile virus. Viruses. 2013;6(1):13-53.

45. Krishnan MN, et al. RNA interference screen for human genes associated with West Nile virus infection. Nature. 2008;455(7210):242-245.

46. Govero J, et al. Zika virus infection damages the testes in mice. Nature. 2016;540(7633):438-442.

47. Miner JJ, et al. Zika virus infection in mice causes panuveitis with shedding of virus in tears. Cell Rep. 2016;16(12):3208-3218.

48. Tabata T, et al. Zika virus targets different primary human placental cells, suggesting two routes for vertical transmission. Cell Host Microbe. 2016;20(2):155-166.

49. Xu Z, Hobman TC. The helicase activity of DDX56 is required for its role in assembly of infectious West Nile virus particles. Virology. 2012;433(1):226-235.

50. Allison SL, Tao YJ, O’Riordain G, Mandl CW, Harrison SC, Heinz FX. Two distinct size class- es of immature and mature subviral particles from tick-borne encephalitis virus. JVirol. 2003;77(21):11357-11366.

51. Liu HM, et al. Regulation of Retinoic Acid Inducible Gene-I (RIG-I) activation by the histone deacetylase 6. EBioMedicine. 2016;9:195-206.

52. Tesh RB, et al. Persistent West Nile virus infection in the golden hamster: studies on its mechanism and possible implications for other flavivirus infections. J Infect Dis. 2005;192(2):287-295.

53. Welte $\mathrm{T}$, et al. $\mathrm{V} \gamma 4^{+} \mathrm{T}$ cells regulate host immune response to West Nile virus infection. FEMS Immunol Med Microbiol. 2011;63(2):183-192.

54. Daffis S, Samuel MA, Suthar MS, Keller BC, Gale $\mathrm{M}$, Diamond MS. Interferon regulatory factor IRF-7 induces the antiviral alpha interferon response and protects against lethal West Nile virus infection. J Virol. 2008;82(17):8465-8475.

55. Ru W, Peng Y, Zhong L, Tang SJ. A role of the mammalian target of rapamycin (mTOR) in glutamate-induced down-regulation of tuberous sclerosis complex proteins 2 (TSC2). J Mol Neurosci. 2012;47(2):340-345.

56. Lanciotti RS, et al. Rapid detection of west nile virus from human clinical specimens, field-collected mosquitoes, and avian samples by a TaqMan reverse transcriptase-PCR assay. JClin Microbiol. 2000;38(11):4066-4071. 